\title{
Langewiesche, Dieter: Vom vielstaatlichen Reich zum föderativen Bundesstaat. Eine andere deutsche Geschichte (Heidelberger Akademische Bibliothek, Bd. 5), 112 S., Kröner Verlag, Stuttgart 2020.
}

\section{Andreas Fahrmeir}

Angenommen: 29. April 2021 / Online publiziert: 22. Mai 2021

(C) Der/die Autor(en) 2021

Dieses Buch ist Teil einer 2019 begründeten Reihe, der Heidelberger Akademischen Bibliothek. Sie ist als Forum gedacht, ,auf dem die Mitglieder der Akademie sich auf eine neue Weise der interessierten Öffentlichkeit vorstellen können“ (S. VIII). Auf den ersten Blick erinnern die Bände freilich an Reihen wie Beck Wissen oder Reclam 100 Seiten: etwas über 100 Seiten in einem handlichen Format, allerdings ästhetisch aufwendiger gestaltet und dadurch weniger günstig. Allerdings ist die Intention eine andere. Soweit sich bislang erkennen lässt, geht es nicht um die Vermittlung von Standardwissen zu mehr oder weniger kanonischen Themen, sondern um akademisch abgewogene, gleichwohl konzise Beiträge zu Fragen, die für aktuelle politische oder kulturelle Debatten von Relevanz sind.

Das trifft, wie die aktuellen Debatten über die Balance zwischen Föderalismus und Zentralisierung, Nationalstaat und Europäisierung überdeutlich machen, für das Thema, das Dieter Langewiesche gewählt hat, in besonderem Maße zu. Zur Geschichte von Nationskonzepten, Nationalismus und Staatlichkeit in Deutschland und Europa hat er bereits zahlreiche grundlegende, konzeptionelle und empirische Werke vorgelegt, aus denen hier hin und wieder auch Passagen aufgegriffen werden; dennoch handelt es sich bei dieser Schrift um ein eigenständig durchkomponiertes Werk.

Es beginnt nach einer Vorstellung unterschiedlicher Konzepte von dem, was eine deutsche Nation sein könnte, mit einem Überblick über Zäsuren der deutschen Geschichte $(1806,1871,1919,1938,1945,1990)$ im europäischen Vergleich, um zu zeigen, dass die politische Organisation Deutschlands in dieser Perspektive Annahmen über eine verspätete Nationsbildung nicht bestätigt, und dass föderale Grundmuster bislang über fast alle Brüche hinweg das zentrale Muster deutscher Geschichte

\footnotetext{
A. Fahrmeir $(\bowtie)$

Goethe-Universität-Frankfurt, Frankfurt a. M., Deutschland

E-Mail: fahrmeir@em.uni-frankfurt.de
} 
darstellten. Die drei folgenden Kapitel vertiefen diese These aus unterschiedlichen Perspektiven mit dem Schwerpunkt auf der Zeit bis zum Ende des wilhelminischen Nationalstaats. Zunächst folgt ein souveräner komprimierter Überblick über die Spannungen zwischen territorialer Herrschaftsentwicklung und übergreifenden Strukturen vom Mittelalter bis zum Deutschen Bund. Sodann geht es um die konkreten politischen Probleme - und ihre Implikationen für die Rolle von deutschen Staaten in einem deutschen Nationalstaat - zwischen 1848 und der Reichsgründung von 1871, wobei vor allem der vergleichende Blick auf Italien gewählt wird. Abschließend richtet sich der Fokus auf die Rolle der internen Pluralität im Kaiserreich, in dem einerseits die Rolle der (nicht von Preußen annektierten) Staaten recht hochgehalten wurde, andererseits (insbesondere vor dem Hintergrund der preußischen Dominanz) eine intensive, vor allem in linken und liberalen Kreisen verbreitete, Kritik entstand, dass der Föderalismus eine Barriere gegen die Parlamentarisierung der Regierung(en) darstelle, die es im Blick zu behalten gelte. Diese Kritik wurde zwar für die Weimarer Republik nicht unmittelbar wirksam, sie hallte aber lange nach, ohne freilich nach den Erfahrungen der NS-Diktatur eine Rückkehr zum Föderalismus, zumindest in Westdeutschland und seit 1990 im wiedervereinigten Deutschland, in Frage zu stellen.

Langewiesches Fazit macht noch einmal deutlich, welchen Beitrag diese Erzählung für gegenwärtige Fragen leisten kann. Sie macht einerseits sichtbar, dass - im europäischen Vergleich - ,zusammengesetzte Staaten' eher die Regel als die Ausnahme waren und dass sich somit Vorstellungen einer, verspäteten Nation', des Föderalismus als Entwicklungshemmnis oder des Nationalstaats als Ziel der Geschichte als zumindest unhistorisch erweisen. Sie macht aber auch deutlich, dass viele der Probleme der Herrschaftsorganisation, die im Rahmen der deutschen Geschichte umstritten waren, sich nun auf europäischer Ebene und mit Blick auf die Beziehungen zwischen der Europäischen Union und den in ihr zusammengeschlossenen Staaten erneut stellen. Dabei betont Langewiesche, dass der Blick in die Geschichte weder Rezepte liefert, noch Muster, die sich zwangsläufig wiederholen, erkennen lässt; aber der Text lenkt sehr gut den Blick auf wahrscheinliche oder naheliegende Wechselwirkungen, die weiterhin zu bedenken sind. Dem Zweck der Reihe, einen abgewogenen und zugleich unmittelbar relevanten Beitrag zu einer längerfristigen Debatte zu liefern, wird der Band also in mustergültiger Weise gerecht.

Funding Open Access funding enabled and organized by Projekt DEAL.

Open Access Dieser Artikel wird unter der Creative Commons Namensnennung 4.0 International Lizenz veröffentlicht, welche die Nutzung, Vervielfältigung, Bearbeitung, Verbreitung und Wiedergabe in jeglichem Medium und Format erlaubt, sofern Sie den/die ursprünglichen Autor(en) und die Quelle ordnungsgemäß nennen, einen Link zur Creative Commons Lizenz beifügen und angeben, ob Änderungen vorgenommen wurden.

Die in diesem Artikel enthaltenen Bilder und sonstiges Drittmaterial unterliegen ebenfalls der genannten Creative Commons Lizenz, sofern sich aus der Abbildungslegende nichts anderes ergibt. Sofern das betreffende Material nicht unter der genannten Creative Commons Lizenz steht und die betreffende Handlung nicht nach gesetzlichen Vorschriften erlaubt ist, ist für die oben aufgeführten Weiterverwendungen des Materials die Einwilligung des jeweiligen Rechteinhabers einzuholen.

Weitere Details zur Lizenz entnehmen Sie bitte der Lizenzinformation auf http://creativecommons.org/ licenses/by/4.0/deed.de. 